lyte concentrations from 34 infants. The results of the sodium and potassium measurements are shown in table 1 and those for $\mathrm{pH}$ and blood gas measurements in table 2.

\section{Discussion}

When sampling blood from neonates it is necessary to keep cumulative blood loss to a minimum by using small sample volumes $(0 \cdot 2 \mathrm{ml}$ in this study), by limiting the number of samples that need to be repeated because of spuriously abnormal results, and by carefully assessing the clinical need for blood analysis before sampling.

The study confirmed our impression that sampling from arterial lines may result in erroneous results and hence possible unnecessary treatment. The underestimation of plasma sodium, potassium, and $\mathrm{PCO}_{2}$ concentrations resulted from dilution of arterial blood with the small volume of $5 \%$ glucose solution in the arterial line. The smaller the flush volume of arterial blood, the greater the dilution. The mixing of $5 \%$ glucose solution (which has a $\mathrm{pH}$ of 5) with arterial blood led to the significantly lower $\mathrm{pH}$ concentrations at flush volumes of $0.5 \mathrm{ml}$.

Concentrations of potassium in capillary blood were higher than those in arterial blood in 38 out of 40 specimens, the mean value being more than $1 \mathrm{mmol}$ higher but with a wide variation (SD 0.96). A high potassium concentration in a capillary sample should be confirmed by measuring the concentrations in a venous or arterial sample, the latter following a $2 \mathrm{ml}$ flush if taken from an indwelling arterial line. There were significant differences between measurements in blood gases in capillary and arterial blood with a moderate rise in carbon dioxide tension in capillary blood and a larger reduction in oxygen tension in capillary blood (mean reduction $2.4 \mathrm{kPa}$ ), but the degree of clinical importance varied unpredictably among patients.

In conclusion, blood samples taken from arterial lines should be preceded by an adequate flush; we regard a $2 \mathrm{ml}$ flush volume as ideal. Capillary blood samples may give erroneously high potassium concentrations even in the absence of obvious haemolysis. Unexpectedly abnormal results should be checked by measuring the concentrations in arterial or free flowing venous blood.

\section{References \\ 1 Wilkinson A, Calvert S. Procedures in neonatal intensive care. In: Roberton NRC, ed. Textbook of neonatology. Edinburgh: Churchill Livingstone, 1986:817-35. \\ 2 Thurlbeck SM, McIntosh N. Preterm blood counts vary with sampling sitc. Arch Dis Child 1987:62:74-5.}

Correspondence to Dr SW Ryan, Department of Paediatrics and Child Health, Clarendon Wing. General Infirmary, Leeds LS2 9NS.

Accepted 14 March 1988

\title{
Cytomegalovirus screened blood for neonatal intensive care units
}

\author{
S LOGAN, ${ }^{*}$ J BARBARA, $\dagger$ AND I KOVAR* \\ ${ }^{*}$ Department of Child Health, Charing Cross and Westminster Medical School, London, and $†$ North London \\ Blood Transfusion Centre, Edgware, Middlesex
}

\begin{abstract}
Summary Two infants were considered to have acquired cytomegalovirus (CMV) infection from blood transfusions screened for the presence of CMV antibody by a haemagglutination assay. Further samples from the same donors were tested by a latex agglutination test; six of $18(33 \%)$ previously believed seronegative were found to be seropositive.
\end{abstract}

The risk of premature infants acquiring cytomegalovirus (CMV) infection from a blood transfusion can be reduced if blood components negative for CMV are used. ${ }^{1}$ In this unit all premature babies receive blood screened for CMV. We describe two cases in which CMV infection occurred despite this policy, and discuss the implications for the provision of blood negative for CMV for neonatal intensive care units.

\section{Case reports}

CASE 1

A female infant was born at 29 weeks' gestation weighing $1200 \mathrm{~g}$. After early problems she progressed well until on day 50 she deteriorated suddenly. Blood cultures grew a group A $\beta$ haemolytic streptococcus. She responded to intravenous antibiotics and was discharged well at 3 months of age.

CMV was isolated from her urine at the time of her deterioration. Routine screening for CMV at birth was negative, and her mother was seronegative for CMV. 
CASE 2

A female infant, the second of twins, was born at 25 weeks' gestation weighing $770 \mathrm{~g}$. She was admitted to the neonatal unit two weeks after case 1 . After a stormy early course she was stable on minimal ventilation by day 25 . On day 32 she deteriorated suddenly with jaundice, hepatosplenomegaly, and thrombocytopenia, and her ventilatory requirements increased. Cerebrospinal fluid and repeated blood cultures were sterile.

CMV was isolated from her urine, tracheal aspirate, and blood and eye swabs. Routine CMV cultures taken at birth were negative. She gradually deteriorated and died aged 148 days. At necropsy CMV inclusion bodies were found in the lung and kidney.

Serum samples taken from her mother at 18 weeks' gestation and during the child's illness showed IgG but no IgM antibody to CMV. The first twin remained well and despite repeated viral cultures CMV was not isolated.

\section{Possible sources of infection}

CMV infection in a neonate may be acquired transplacentally, ${ }^{2}$ during passage through the birth canal, ${ }^{3}$ from breast milk, ${ }^{3}$ from blood components, ${ }^{1}$ or possibly by horizontal spread. ${ }^{4}$ In order to exclude the possibility of horizontal spread from one of these infants to the other, virus isolates were typed using DNA restriction endonuclease analysis 5 and found to be dissimilar.

In case 1 the mother was seronegative and could not have been the source of infection. The child received breast milk only from her mother. In case 2 congenital CMV had been excluded. The infant received only parenteral nutrition. Infection during delivery was a possible source, although the other twin was not infected. Unfortunately it was not possible to take cervical or urine samples from the mother for viral studies.

Both affected infants had received multiple blood transfusions. The blood had been screened for the presence of CMV antibodies using a dilution modification of a haemagglutination assay (Cetus Corporation, subsequently withdrawn). As reported elsewhere, ${ }^{6}$ concerns about consistency within batches and the availability of a more convenient assay had already lead to the introduction of a modified latex agglutination test (Becton Dickinson) as the preferred screening test by the blood transfusion centre.

Repeat blood samples from the donors whose blood had been given to these infants were tested for the presence of CMV antibody using the more sensitive latex agglutination test. Case 1 received
Table Comparison of CMV serology on repeat samples

\begin{tabular}{llll}
\hline & \multicolumn{2}{l}{ First test (haemagglutination assay) } \\
\cline { 2 - 4 } & No positive & No negative & Total No \\
\hline $\begin{array}{l}\text { Second test (latex } \\
\text { agglutination test): } \\
\quad \text { No positive }\end{array}$ & 0 & & \\
$\quad$ No negative & 1 & 12 & 6 \\
\hline Total No & 1 & 18 & 13 \\
\hline
\end{tabular}

blood from six donors, five of whom provided repeat samples; one was found to be positive for CMV by the latex agglutination test. Case 2 received 15 transfusions. Blood samples were obtained from 14 of the donors; five were seropositive by the latex agglutination test. One unit, used under the mistaken impression that it was seronegative, was found on rechecking to have been positive on the initial haemagglutination assay. A repeat sample from the donor, however, was seronegative by the latex agglutination test. This unit was transfused after the child acquired CMV infection.

Overall, of the 18 donors found negative on the original assay, six $(33 \%)$ were seropositive by the latex agglutination method (table).

\section{Discussion}

Failure of a test may be the result of inherent problems in the test or of human error. The haemagglutination assay was widely used and generally regarded as reliable and sensitive. Comparison with a radioimmunoassay, however, suggested that false negative results were occurring and this led to the introduction of the latex agglutination test. ${ }^{6}$ Human error was the probable reason for the incorrect use of one unit after a positive (although probably false positive) test result.

The regional blood transfusion centre screens up to 200 units each day for the presence of CMV antibodies. Much of the demand for screened blood comes from neonatal units. When we checked some of the requests there seemed to be confusion among clinicians about the indications for the use of blood negative for CMV. Yeager et al showed no appreciable morbidity associated with the use of unscreened blood in babies of more than 28 weeks' gestation nor in those born to seropositive mothers. They therefore recommended that blood negative for CMV should be used only for babies of less than 32 weeks' gestation born to seronegative mothers.

The mother of our child who died was seropositive; we believe that if the baby is extremely premature the restriction of screened blood to the 
offspring of seronegative mothers may not be appropriate. There is, however, no evidence that babies of more than 32 weeks' gestation benefit from the use of blood negative for CMV. Using gestational age rather than birth weight as the criterion for the use of blood screened for CMV seems reasonable, as morbidity from neonatal CMV infection is thought to be related to immaturity of the immune system.

Although the blood transfusion services are responsible for continuously monitoring the reliability of their test procedures, clinicians must take some responsibility. Firstly, laboratory evaluation of tests does not replace clinical observation. Any clinician who believes that a child has acquired CMV infection from blood products should discuss this with the blood transfusion service so that further investigation may be undertaken. Secondly, by limiting the use of specialised services such as the provision of blood screened for CMV to those cases for whom it is essential, the load on the service can be decreased and its efficiency increased.
References

1 Yeager AS, Grumet FC, Hafleigh EB, Arvin AM, Bradley JS, Prober CG. Prevention of transfusion-acquired cytomegalovirus infections in newborn infants. J Pediatr 1981;98:281-7.

2 Peckham CS, Chin KS, Coleman JC, Henderson K, Hurley R, Preece PM. Cytomegalovirus infection in pregnancy: preliminary findings from a prospective study. Lancet 1983;i:352-5.

${ }^{3}$ Stagno S, Pass RF, Dworsky ME, Alford CA Jr. Maternal cytomegalovirus infection and perinatal transmission. Clin Obstet Gynecol 1982;25:563-76.

4 Spector SA. Transmission of cytomegalovirus among infants in hospital documented by restriction-endonuclease-digestion analysis. Lancet 1983;i:378-81.

5 Huang ES, Kilpatrick BA, Huang YT, Pagano JS. Detection of human cytomegalovirus and analysis of strain variation. Yale $J$ Biol Med 1976;49:29-43.

6 Barbara JAJ, Moulsdale H, Brown S, Griffiths PD, Berry MJ, Contreras M. Modified latex agglutination test for anticytomegalovirus, suitable for pretransfusion screening. $J$ Clin Pathol 1987;40:115-6.

Correspondence to Dr S Logan, Department of Epidemiology, Institute of Child Health, 30 Guilford Street, London WC1N 1EH.

Accepted 19 January 1988

\title{
Reduction of head flattening in preterm infants
}

\author{
P H T CARTLIDGE AND N RUTTER
}

Department of Neonatal Medicine and Surgery, City Hospital, Nottingham

SUMmary During the first few weeks of life many preterm infants develop flattened heads. We have shown that this deformity can be reduced by nursing preterm infants on soft, air filled mattresses of the type used for detecting apnoea.

The shape of a newborn infant's head is independent of gestation because the support given by amniotic fluid is uniform and allows unrestrained growth. ${ }^{1}$ After birth, however, more localised pressures are encountered and side to side flattening of the head may occur because infants are usually nursed with their heads turned laterally. ${ }^{12}$ In infants born at term the effect is mild, but in preterm infants the deformity is often pronounced and may persist into adulthood. ' This is probably because preterm infants have thin, soft skull bones that are more malleable. In addition, they spend many weeks lying only on the sides of the head until they become developmentally mature enough to adjust their position voluntarily.

Head flattening has two clinical consequences.
Firstly, it may result in misleadingly high measurements of occipitofrontal circumference because for a given area the perimeter of an ellipse is greater than that of a circle. ${ }^{1}$ Secondly, from a cosmetic point of view parents prefer their child to have a normally shaped head. ${ }^{3}$ In this study we have investigated whether the deformation can be reduced by nursing preterm infants on soft, air filled mattresses.

\section{Subjects and methods}

Infants of less than 32 weeks' gestation born at this hospital were included in the study. Within 24 hours of birth they were alternately assigned to be nursed either on a standard, firm, foam filled mattress 12 $\mathrm{mm}$ thick supplied with Vickers 79 incubators, or on a soft, air filled mattress. The air mattress was of the type used to detect apnoea (Apnoea alarm Mark 3, Vickers Medical, Hampshire), and was inflated to a pressure of about $2 \mathrm{~cm} \mathrm{H}_{2} \mathrm{O}$. Apnoea in infants nursed on the standard mattress was detected by a respiratory sensor taped to the abdomen.

Measurements to determine head shape were made by a single observer (PHTC). The occipito- 